\title{
metaX: a flexible and comprehensive software for processing metabolomics data

Bo Wen ${ }^{1,2}$, Zhanlong Mei ${ }^{1,2}$, Chunwei Zeng ${ }^{1,2}$ and Siqi Liu ${ }^{1,2^{*}}$

\begin{abstract}
Background: Non-targeted metabolomics based on mass spectrometry enables high-throughput profiling of the metabolites in a biological sample. The large amount of data generated from mass spectrometry requires intensive computational processing for annotation of mass spectra and identification of metabolites. Computational analysis tools that are fully integrated with multiple functions and are easily operated by users who lack extensive knowledge in programing are needed in this research field.

Results: We herein developed an R package, metaX, that is capable of end-to-end metabolomics data analysis through a set of interchangeable modules. Specifically, metaX provides several functions, such as peak picking and annotation, data quality assessment, missing value imputation, data normalization, univariate and multivariate statistics, power analysis and sample size estimation, receiver operating characteristic analysis, biomarker selection, pathway annotation, correlation network analysis, and metabolite identification. In addition, metaX offers a webbased interface (http://metax.genomics.cn) for data quality assessment and normalization method evaluation, and it generates an HTML-based report with a visualized interface. The metaX utilities were demonstrated with a published metabolomics dataset on a large scale. The software is available for operation as either a web-based graphical user interface (GUI) or in the form of command line functions. The package and the example reports are available at http://metax.genomics.cn/.
\end{abstract}

Conclusions: The pipeline of metaX is platform-independent and is easy to use for analysis of metabolomics data generated from mass spectrometry.

Keywords: Metabolomics, Pipeline, Workflow, Quality control, Normalization

\section{Background}

Biochemicals (metabolites) with low molecular masses are the ultimate products of biological metabolism, while a metabolome represents the total composite in a given biological system and reflects the interactions among an organism's genome, gene expression status and the relevant micro-environment [1]. The most prevalent technology used in analysis of metabolomics is non-targeted mass spectrometry (MS) coupled with either liquid chromatography (LC-MS) or gas chromatography (GC-MS) [2,3]. Generally, these techniques generate a set data of mass spectra with chromatography that includes retention time, peak intensity and chemical masses. Data analysis involves stepwise

\footnotetext{
* Correspondence: siqiliu@genomics.cn
'BGI-Shenzhen, Shenzhen 518083, China

* Correspondence: siqiliu@genomics.cn
${ }^{1}$ BGI-Shenzhen, Shenzhen 518083, China

${ }^{2}$ China National GeneBank-Shenzhen, BGI-Shenzhen, Shenzhen, Guangdong

518083, China
}

procedures including peak picking, quality control, data cleaning, preprocessing, univariate and multivariate statistical analysis and data visualization. A number of software packages are available for MS-based metabolomics data analysis as listed in Table 1, including propriety commercial, open-source, and online workflows. The MS manufacturers generally provide propriety software, like SIEVE (Thermo Scientific), MassHunter (Agilent Technologies) and Progenesis QI (Waters), which are often limited in scope and function. Open-source software, such as XCMS [4], CAMERA [5], MAIT [6], MetaboAnalyst [7] and Workflow4Metabolomics [8], usually cover limited processing steps. There is no such comprehensive pipeline that is used across the metabolomics commity $[9,10]$. Referring to the capabilities of the tools mainly used (as shown in Table 1), an automatic and comprehensive open source pipeline is urgent in 
Wen et al. BMC Bioinformatics (2017) 18:183

Page 2 of 14

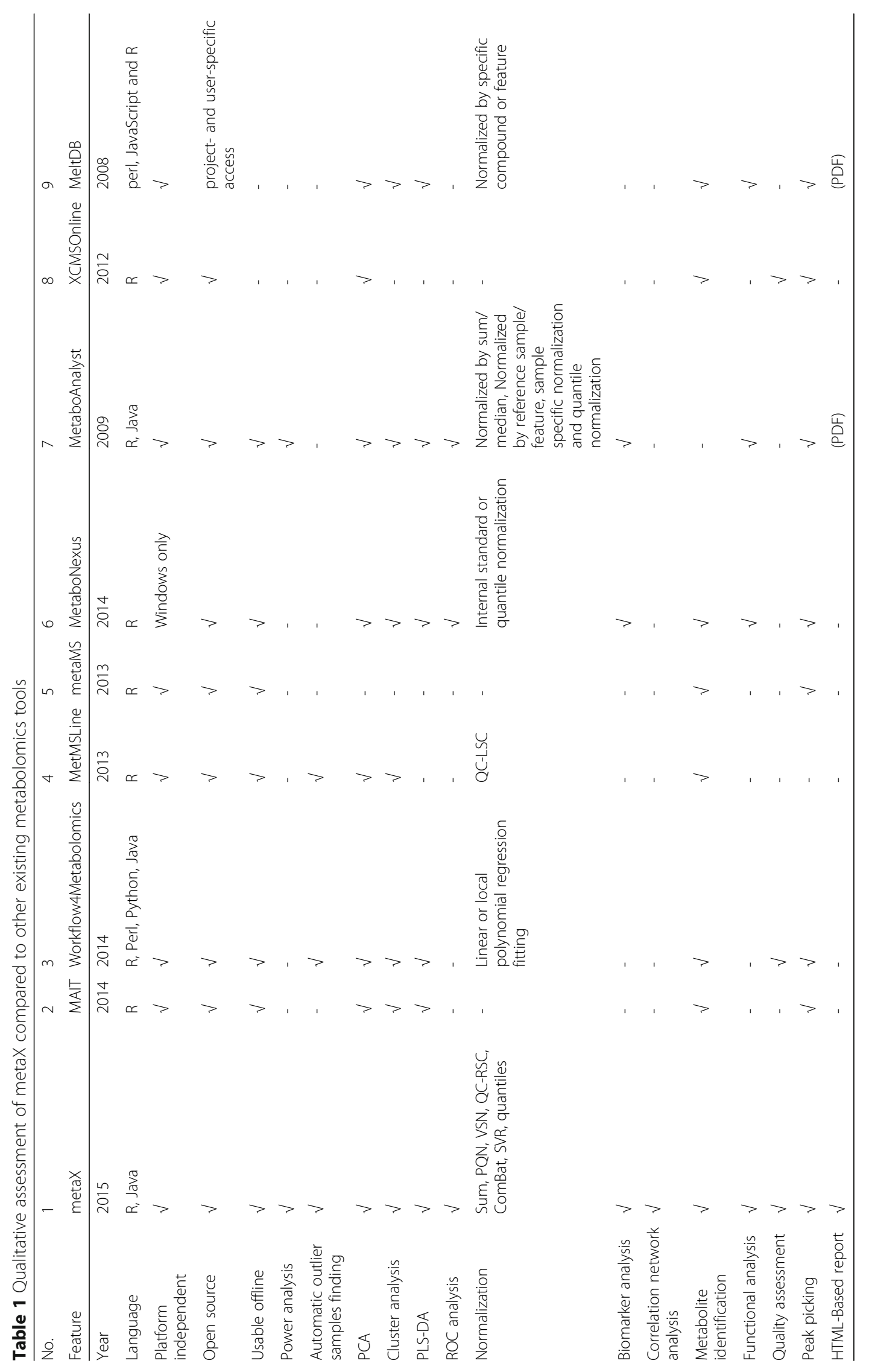


Wen et al. BMC Bioinformatics (2017) 18:183

Page 3 of 14

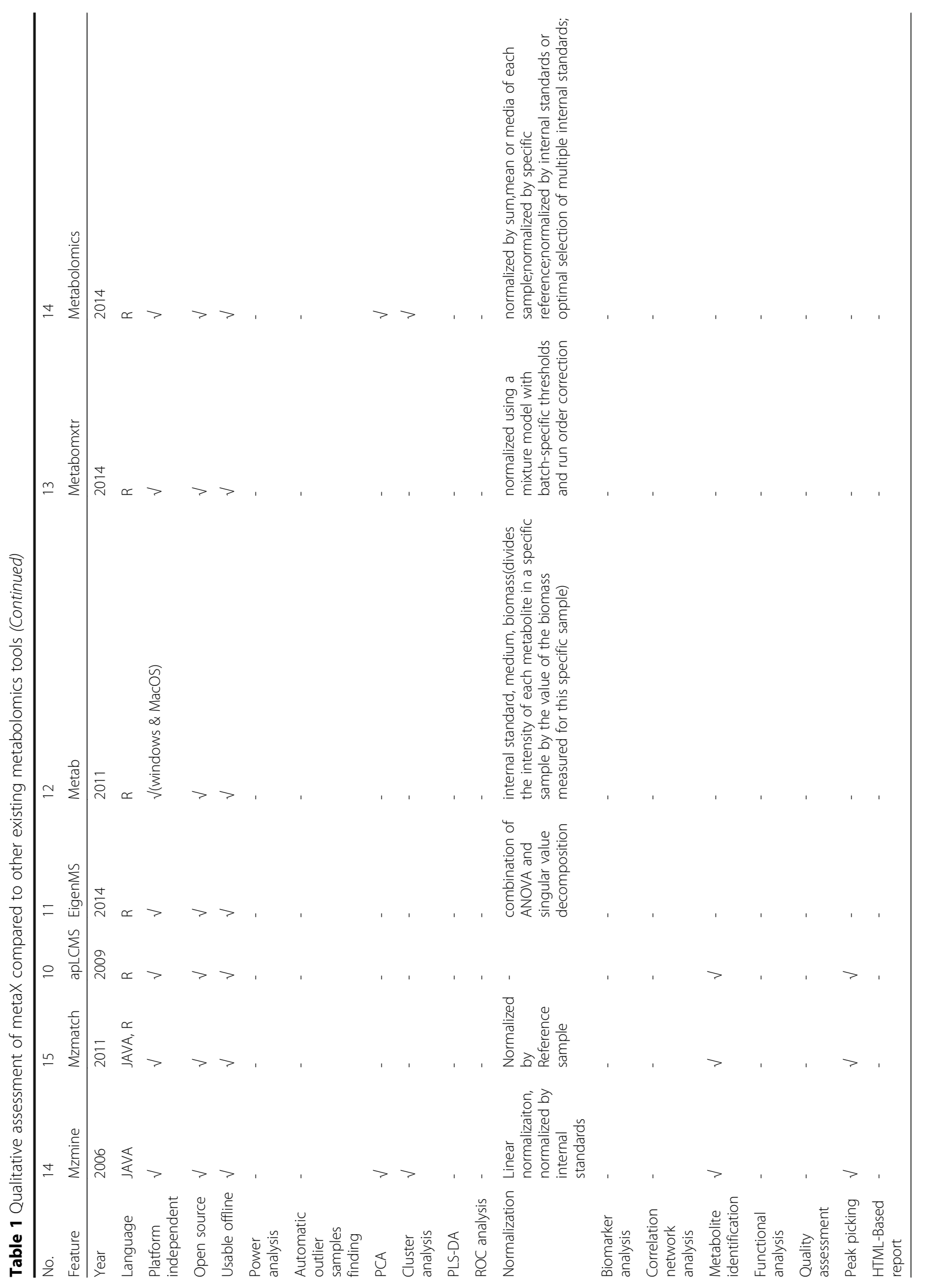


bioinformatics analysis of metabolomics. Basically, the pipeline aims for users to easily perform end-to-end metabolomics data analysis with a flexible combination of different methods to efficiently integrate new modules and to build customized pipelines in multiple ways.

We herein developed a comprehensive workflow for analysis of metabolomics data, termed metaX. At the present time, $R$ [11] is a popular statistical programming environment and provides a convenient environment for statistical analysis of metabolomic and other -omics data [12,13]. We thus designed metaX as an $\mathrm{R}$ package that automates analysis of untargeted metabolomics data acquired from LC/MS or GC/MS and offers a user-friendly web-based interface for data quality assessment and normalization evaluation. This workflow, which is open source and rich in functions, encourages experienced programmers to improve the relevant functions or to build their own pipeline within the $\mathrm{R}$ framework. Overall, metaX aims to be a tool array that utilizes an end-to-end statistical analysis of metabolomics data.

\section{Implementation}

A stepwise overview of data processing using metaX is illustrated in Fig. 1.

\section{Peak picking and inputs}

In general, metaX can take mzXML files as input or a peak table file as input. If taking mzXML files as input, metaX will use the R package XCMS [4] to detect peaks, then use the CAMERA [5] package to perform peak annotation. If a peaks table file is an input, metaX transforms the table data from a peak detection software, such as Progenesis QI (exported comma separated value (csv) format file), into an $\mathrm{R}$ object compatible with the subsequent workflow.

\section{Pre-processing of raw peak data metabolite}

The raw peak intensity data was pre-processed in metaX. Firstly, if a metabolite feature is detected in $<50 \%$ of quality control (QC) samples or detected in $<20 \%$ of experimental samples, it is removed from data analysis [14]. Secondly, a missing value after the first filtering is retained and imputed. In metaX, four methods are implemented to perform missing value imputation: $k$ nearest neighbor (KNN), Bayesian principal component analysis replacement (BPCA), svdImpute and random forest imputation (missForest) [15].

\section{Data scaling and transformation}

Five different scaling approaches are offered in metaX: Pareto scaling, vast scaling, range scaling, autoscaling

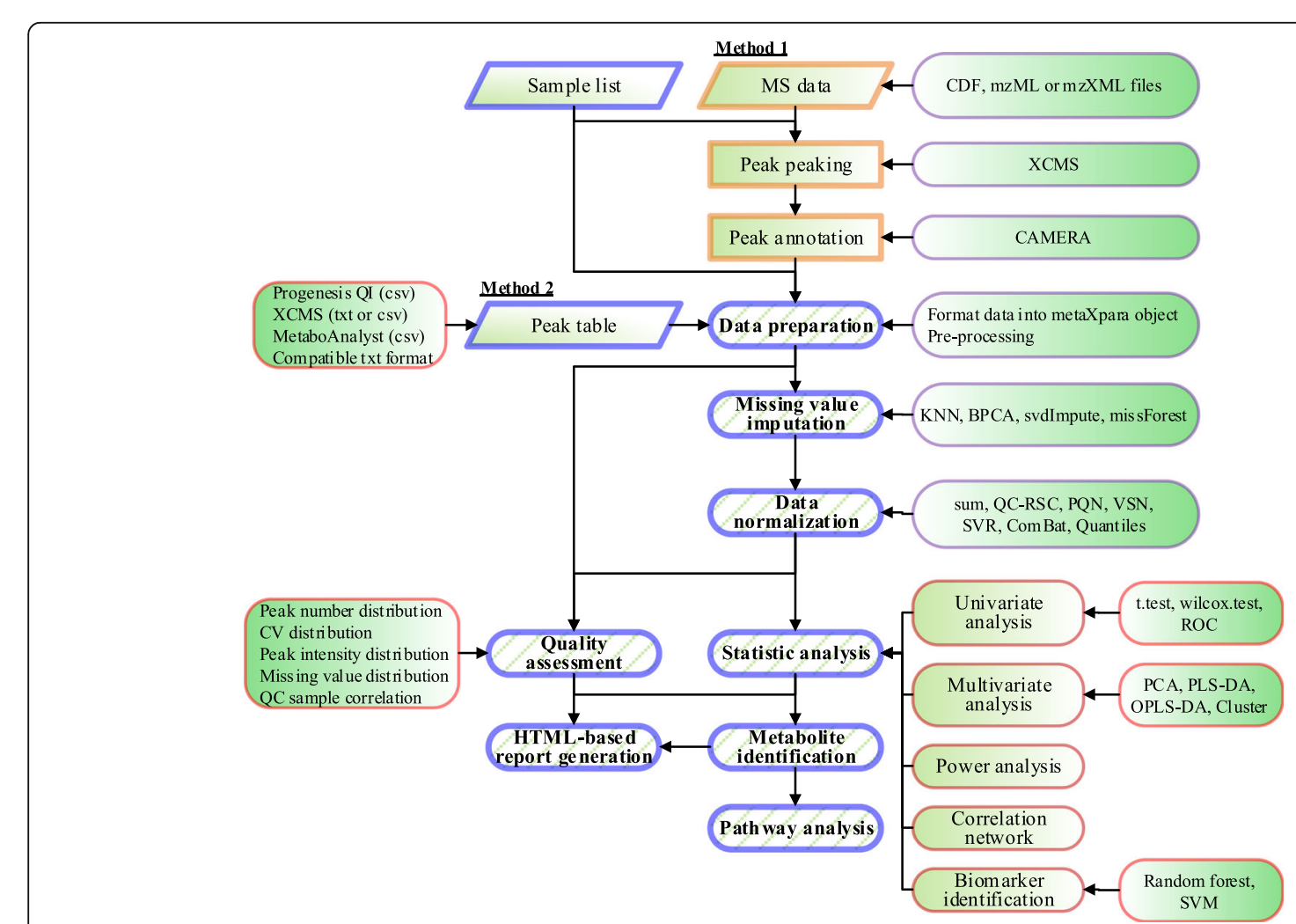

Fig. 1 Overview of metaX. This figure summarizes the main modules, functions and features of metaX. The input data and the functions are included in the figure 
and level scaling [16]. The formulas of these scaling approaches are described in detail elsewhere [16]. In addition, three transformation approaches are offered in metaX: log, generalized logarithm (glog) and cube root transformation.

\section{Removal of outliers}

metaX provides the ability to automatically remove the outlier samples in the pre-processed data based on expansion of the Hotelling's T2 distribution ellipse [17]. A sample within the first and second component principal component analysis (PCA) score plot beyond the expanded ellipse is removed, and then the PCA model is recalculated. In default mode, three rounds of outlier removal are performed.

\section{Normalization}

A metabolomics dataset usually contains unwanted variations introduced by signal drift/attenuation and multiplicative noise across the dynamic range. These effects can detrimentally impact the significant signal discovery and MS features that are required for rigorous quality assurance $[14,18]$. In metaX, two types of normalization methods are provided: 1) Sample-based normalization is used to correct different concentrations of samples, such as normalization to total sum, probabilistic quotient normalization (PQN), variance stabilizing normalization (VSN) and quantilebased methods. 2) Peak-based normalization is implemented to correct data within batch experiment analytical variation and batch-to-batch variation in large-scale studies [19]. In this normalization, if a study contains QC samples, the QC-robust spline batch correction (QC-RSC) can be used to alleviate the effects of peak area attenuation [19]. During normalization, the degree of smoothening is controlled by a parameter that sets the proportion of points for smoothening at each point, while in metaX, this parameter is automatically assigned by using leave-one-out cross validation. On the basis of QC samples, a metabolite feature with a coefficient of variation $(\mathrm{CV})$ over the predetermined value is excluded after normalization. The CV threshold could be set by users; generally, CV values $\leq 30 \%$ are recommended. Support vector regression (SVR) [20] and ComBat [21] normalization methods are also implemented in metaX. A user-friendly web-based interface (http:// metax.genomics.cn) was offered for rapid evaluation of the data normalization methods for a specified dataset.

\section{Assessment of data quality}

Pre- and post-normalization, the data quality is visually assessed in several aspects, 1) the peak number distribution, 2) the number of missing value distribution, 3) the boxplot of peak intensity, 4) the total peak intensity distribution, 5) the correlation heatmap of QC samples if available, 6) the metabolite $\mathrm{m} / \mathrm{z}$ (or mass) distribution, 7) the plot of $\mathrm{m} / \mathrm{z}$ versus retention time, and 8) the PCA score or loading plot of all samples. There are two ways to perform data quality assessment in metaX, the command line mode and the user-friendly web-based interface at http://metax.genomics.cn/.

\section{Univariate and multivariate statistical analysis}

metaX offers both univariate and multivariate statistical analysis. For univariate statistical analysis, the parametric statistical test (Students t-test), non-parametric statistical test (Mann-Whitney $\mathrm{U}$ test), and classical univariate receiver operating characteristic (ROC) curve analysis are implemented. For multivariate statistical analysis, metaX offers functionalities for cluster analysis, multivariate modelling, including PCA, partial least squares-discriminant analysis (PLS-DA) and orthogonal partial least squaresdiscriminant analysis (OPLS-DA), with numerical and graphical results and diagnostics (optimal number of components estimated by cross-validation, $\mathrm{R}^{2}, \mathrm{Q}^{2}$, variable importance in projection (VIP), statistical significance of the model by permutation testing) [22]. In terms of the univariate test analysis, metaX also offers the false discovery rate (FDR)-corrected $p$-value by using the Benjamini-Hochberg FDR algorithm [23]. The PLS-DA was implemented based on the functions from the pls package [24], and the OPLSDA was performed using the functions from the ropls package [25].

\section{Power and sample size analysis}

metaX offers an easy-to-use function to perform the power and sample size analysis. This function is based on the Bioconductor package SSPA [26] and outputs a figure to show the distribution curve of sample size versus the estimated power.

\section{Metabolite correlation network analysis}

metaX offers two types of network analysis. One is the correlation network analysis without regard for experimental groups information, and the other is differential correlation network analysis, which aims to identify metabolite correlation differences in a physiological state. The former was implemented using the cor function from the stats package to calculate the correlation coefficient, and the latter was implemented using the function comp.2.cc.fdr from the DiffCorr package [27] to calculate the significantly differential correlations. The igraph package [28] was used for network analysis and visualization. In addition, the network can be exported as a file in formats such as gml and pajek, which can be imported into Cytoscape [29] and Gephi [30] for network analysis and visualization. Both of the correlation network analyses aim to describe the correlation patterns among metabolites across samples, in which nodes represent metabolites and edges represent the correlation between different metabolites. The network analysis offers 


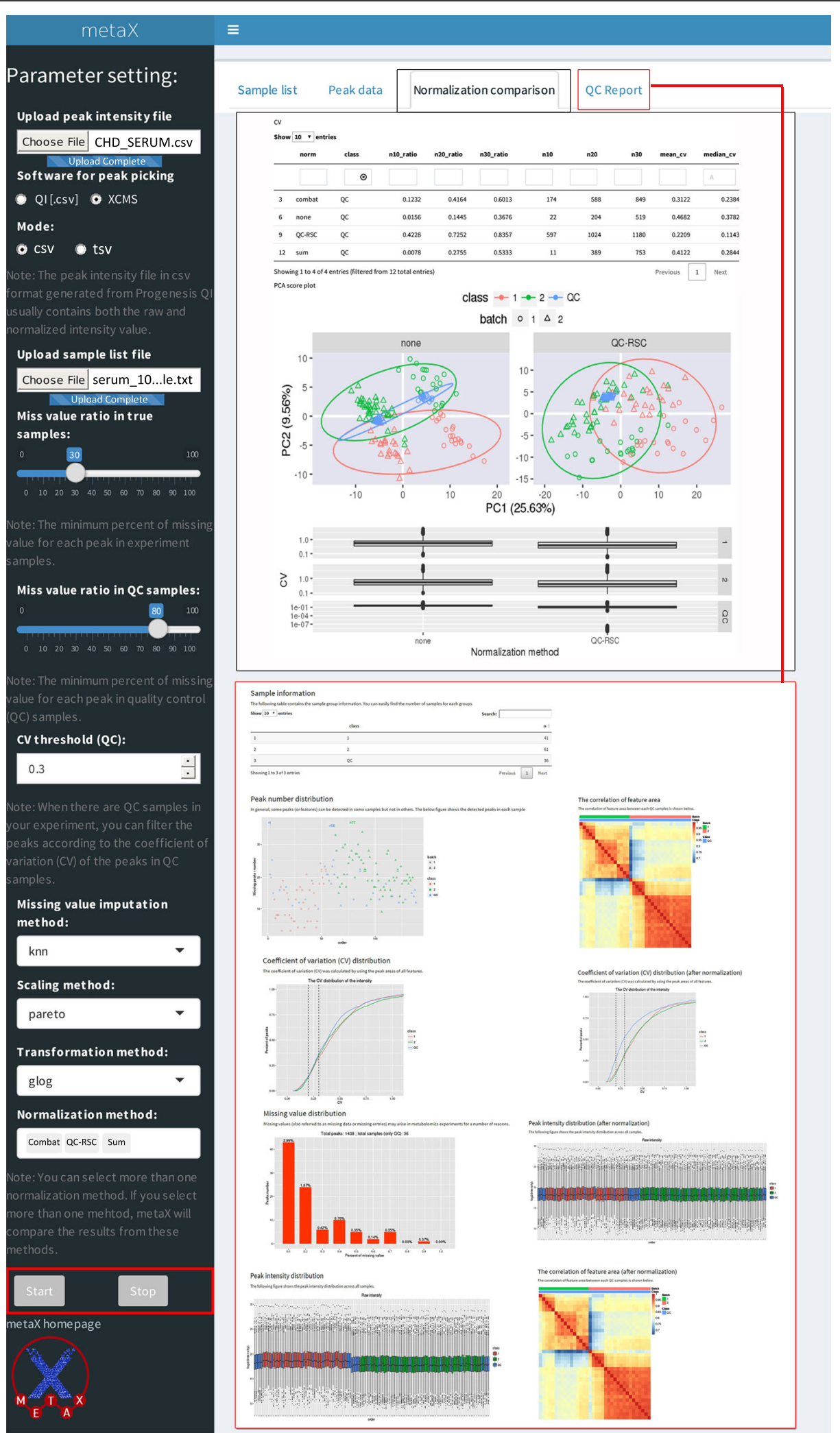

Fig. 2 User interface of metaX for quality assessment and normalization evaluation 
a complementary method to univariate and multivariate statistical analysis methods.

\section{Metabolite identification}

Currently, metaX provides a function for metabolite identification based on the Human Metabolome Database (HMDB) [31], KEGG [32, 33], MassBank [34], PubChem [35], LIPID MAPS [36], MetaCyc [37] and PlantCyc (www.plantcyc.org). Moreover, metaX can easily be extended to support the other databases. The metabolites having molecular weights within a specified tolerance to the query $\mathrm{m} / \mathrm{z}$ or molecular weight value are retrieved from the databases as putative identifications. The information of adducts and isotopes is utilized to assist in metabolite identification if it is present. The default tolerance is $10 \mathrm{ppm}$.

\section{Functional analysis}

At present, metaX provides a function for metabolite pathway analysis based on IMPaLA [38].

\section{Biomarker analysis}

metaX uses functions from the $\mathrm{R}$ package "caret" to perform the biomarker selection, model creation and performance evaluation [39]. Currently, two methods, random forest [40] and support vector machine (SVM), are implemented to automatically select the metabolites which show the best performance. After the best set features are

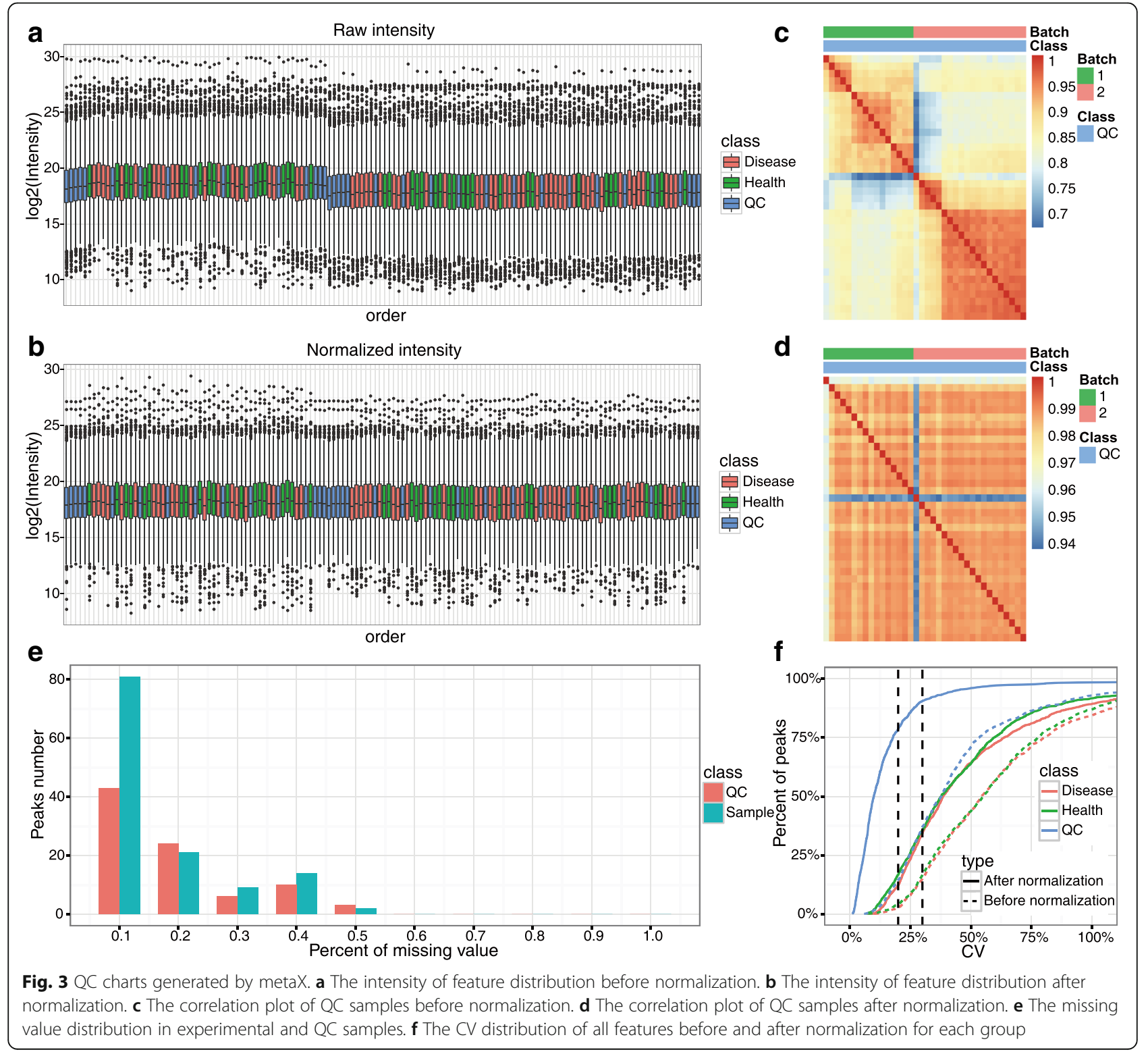


selected, a randomForest model can be created and the ROC curve can be plotted.

\section{HTML-based report generation}

metaX outputs an HTML-based report by using the Nozzle package [41], which contains quality assessment plots and other analysis results.

\section{Results and discussion}

To illustrate the applications of metaX, a published nontargeted LC-MS metabolomics dataset from a coronary heart disease (CHD) study was used [42, 43]. The dataset consisted of two batches of 138 plasma samples (59 CHD patients, 43 healthy controls and $36 \mathrm{QC}$ samples) acquired in positive ion mode on an LTQ Orbitrap Velos instrument (Thermo Fisher Scientific, MA, USA). LC-MS raw data files were converted to mzXML format using ProteoWizard (version 3.0.5941) [44] and then were processed by XCMS [4] and CAMERA [5] for peak picking and peak annotation, respectively. In total, 1438 features were retained for downstream analysis. The mzXML files can be downloaded from the Dryad Digital Repository [43]. It merits to note that the study focus is mainly on the software application and its capabilities, not on the biological interpretation of the generated results.

\section{Quality assessment of metabolomics data using metaX}

In metabolomics studies, data quality checks are crucial prerequisites to achieve reliable results. metaX offers a quick and easy data quality check of metabolomics data. This can be done using the $\mathrm{R}$ function in metaX or a userfriendly web interface at the website http://metax.genomics.cn/ as shown in Fig. 2. The mainly QC charts generated by metaX for the CHD dataset are illustrated in Figs. 3 and 4. The number of features detected per sample over the analysis time (injection order) is illustrated in Fig. 4c, revealing that the peaks acquired from any group, disease, healthy and $\mathrm{QC}$, are randomly distributed. The intensities of all features per samples before and after normalization over the analysis time (injection order) are illustrated in Fig. 3a and b, respectively. The missing value distribution is shown in Fig. 3e, which gives an overview of the percent of missing values of all features in both the QC and experiment samples. According to Chawade's view, the total missing value plot and the total intensity plot derived from raw data and treated with/without normalization

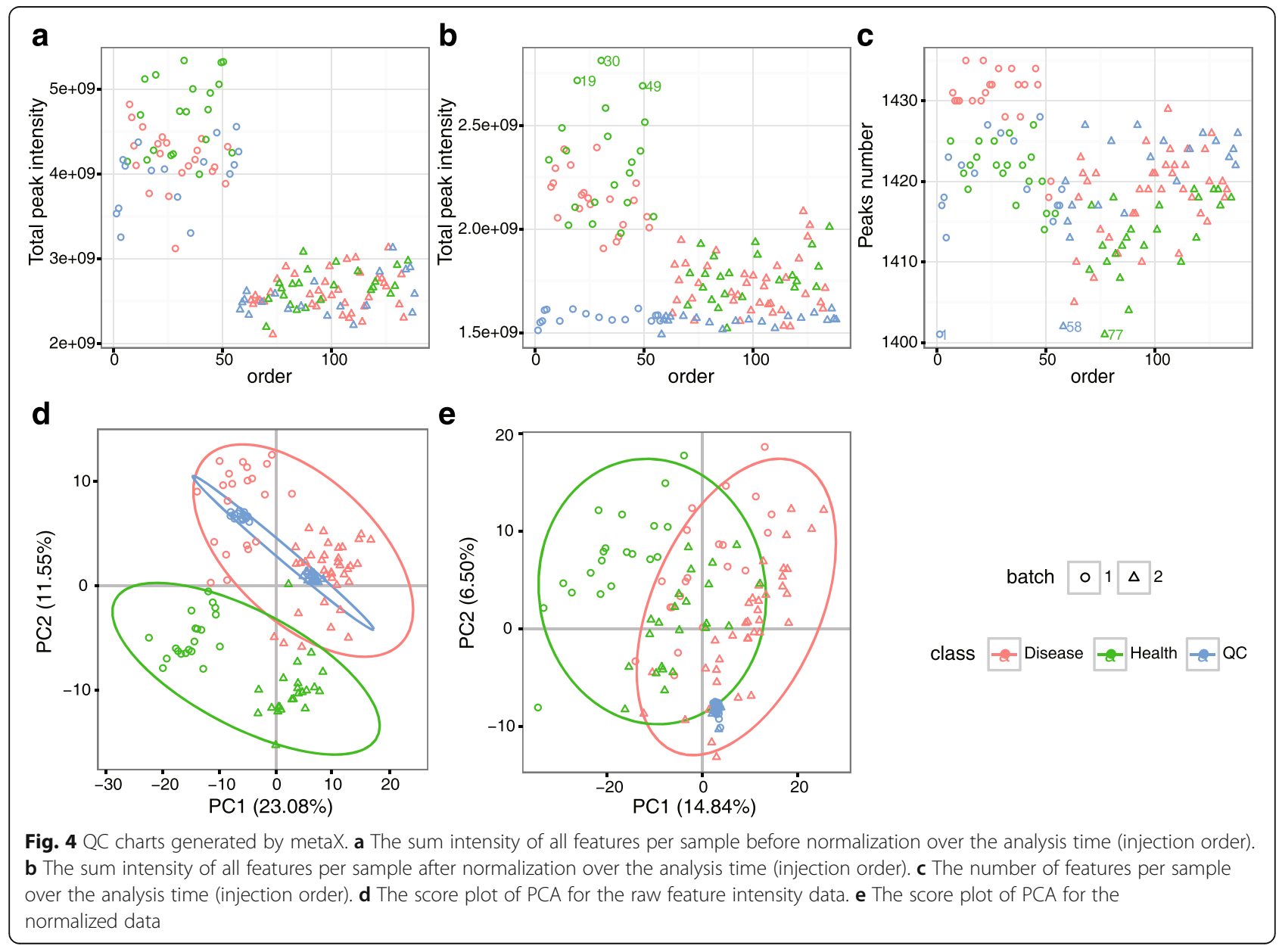


could be used to identify sample outliers [45]. Our analysis supported this. The correlation plots of QC samples before and after normalization by SVR are illustrated in Fig. 3c and $\mathrm{d}$ and indicate that the lowest correlation efficiency is enhanced from approximately 0.7 to 0.9 . The $\mathrm{CV}$ distribution of all features before and after normalization for each group is displayed in Fig. 3f, implying that after normalization, the signal quality is obviously improved. The sum intensity of all features per sample before and after normalization over the analysis time (injection order) is illustrated in Fig. 4a and b, suggesting that normalization could narrow the signal variation. The score plots of PCA for the raw feature intensity data and the normalized data are shown in Fig. 4d and e, respectively, which give an overview of the dataset and showing trends, groupings and outliers before data normalization and after data normalization. The score plot of PCA (Fig. 4d) for the nonnormalized data provided a simple and easily interpretable visual check of the presence of batch effects. In Fig. 4d, the two data batches appear as two separated groups upon PCA analysis without normalization, whereas in Fig. 4e, after normalization the batch effect was reduced and all of the QC samples were clustered tightly, which provides an initial evaluation of the data quality. Overall, these QC charts demonstrate the necessity of normalization for metabolomics data, while metaX enables overview of the data quality with different charts.

\section{Evaluation of normalization methods using metaX}

A systematic bias in high-throughput metabolomics data is often introduced by various steps of sample processing and data generation. Data normalization can reduce systematic biases. A question related to this issue is how to select a proper normalization method. metaX provides a user-friendly web-based Shiny application (http://metax. genomics.cn) for this purpose. To select the optimal normalization approach for the CHD dataset, seven methods are evaluated using metaX. Figure 5 shows the score plots of PCA using different normalization methods. They indicate that after normalization using QC-RSC, ComBat or SVR, all of the QC samples are clustered more tightly, and the batch effect is effectively reduced compared with other methods. Table 2 presents the quantitative comparison metrics acquired by the different methods. From the results it is clear that all normalization methods performed better than nonnormalization used in most of the metrics. Specifically, SVR detects the largest number of features (1293) with $\mathrm{CV} \leq 30 \%$ in QC samples, followed by QC-RSC (1191). For the average $\mathrm{CV}$ of features in QC samples, SVR achieved the best performance, followed by QC-RSC. This is similar to the findings in a previous study [20]. However, QC-RSC could detect the largest number of differentially expressed features (178), followed by SVR (170). Taken together, for this data set, SVR could be an

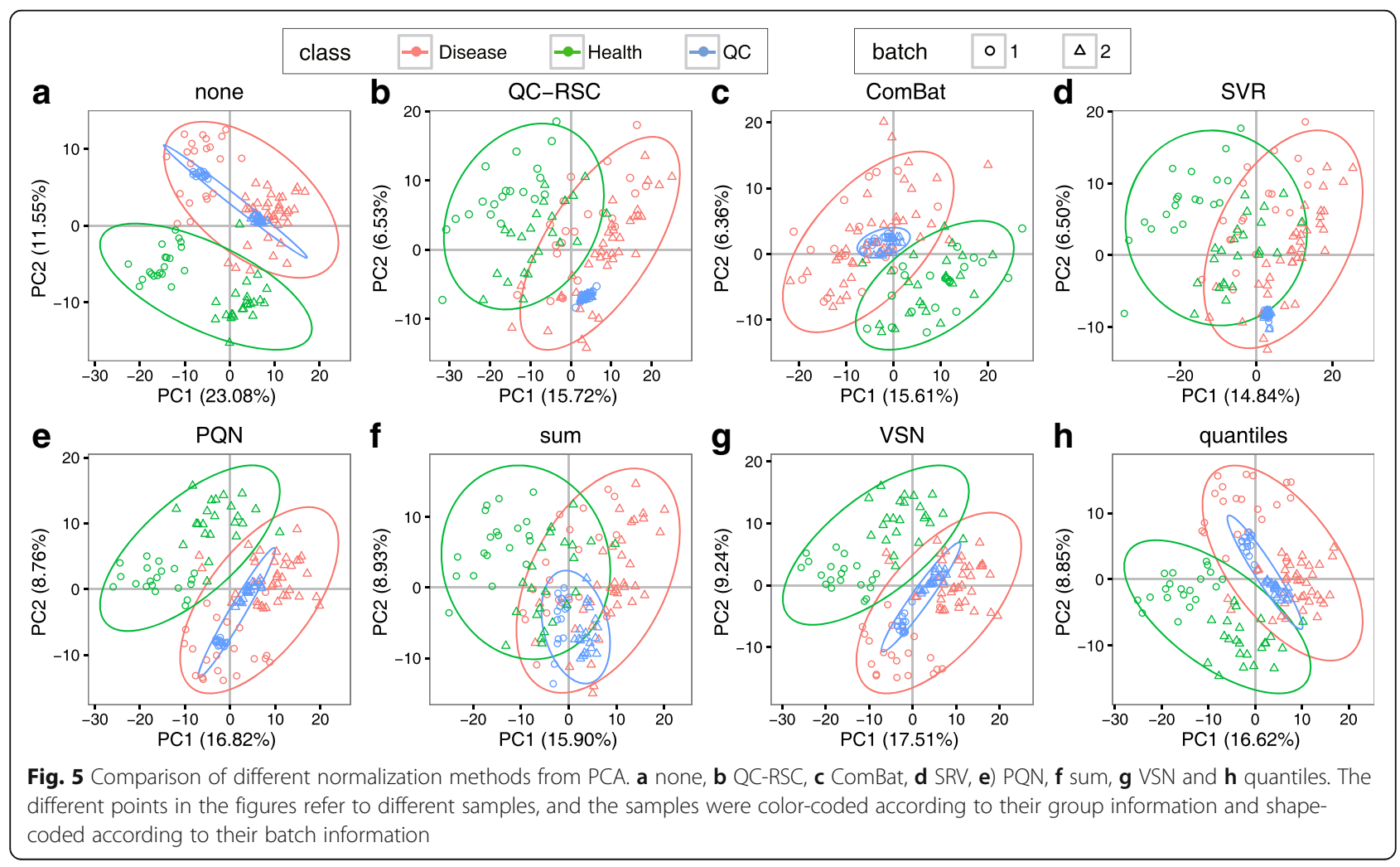


Table 2 The comparison of different normalization methods

\begin{tabular}{|c|c|c|c|c|c|c|}
\hline Methods & NO. of peaks & NO. of peaks $(C V \leq 30 \%)^{a}$ & $\mathrm{DEF}^{\mathrm{b}}$ & Mean $(\mathrm{CV})^{\mathrm{CHD} d}$ & Mean (CV) Health $d$ & Mean $(\mathrm{CV})$ QC e \\
\hline ComBat & 1438 & 930 & 127 & 0.4261 & 0.3816 & 0.1636 \\
\hline none & 1438 & 527 & 65 & 0.4865 & 0.4739 & 0.2114 \\
\hline QC_RSC & 1438 & 1191 & 178 & 0.5108 & 0.4664 & 0.1098 \\
\hline SVR & 1438 & 1293 & 170 & 0.4853 & 0.4583 & 0.1081 \\
\hline PQN & 1438 & 793 & 125 & 0.4945 & 0.4681 & 0.1777 \\
\hline Quantiles & 1438 & 740 & 118 & 0.4911 & 0.4646 & 0.1895 \\
\hline sum & 1438 & 761 & 119 & 0.5044 & 0.4733 & 0.1979 \\
\hline VSN & 1438 & 772 & 120 & 0.5014 & 0.4761 & 0.1912 \\
\hline
\end{tabular}

Note:

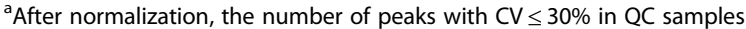

${ }^{b} \mathrm{DEF}$ : differentially expressed features with $q$-value $<=0.05$, fold change $>=1.5$ or fold change $<=0.667$ and VIP $>=1$

${ }^{\mathrm{C}}$ Mean (CV) ${ }^{\mathrm{CHD}}$ : The average CV of peaks in CHD disease group

${ }^{\mathrm{d}}$ Mean (CV) Health: The average CV of peaks in health group

${ }^{\mathrm{e}}$ Mean (CV) ${ }^{\mathrm{QC}}$ : The average CV of peaks in QC group

optimal normalization method, thus it was chosen as the default normalization method for the downstream analysis.

\section{Univariate and multivariate statistical analysis}

Data for the QC samples are removed from the dataset prior to univariate and multivariate analysis in metaX. For univariate analysis, Mann-Whitney U test and Students t-test are performed to compare disease and health groups, followed by false discovery correction using the Benjamini-Hochberg method using metaX. The results, along with the fold change of the disease group versus health group, are presented in Additional file 1: Table S1. In total, 171 features (13.22\% of total features) are detected under the criteria of the corrected $p$-value (Mann-Whitney $U$ test) $\leq 0.05$, fold change $\geq 1.5$ or $\leq$ 0.667 and VIP $>=1$, and 170 features $(13.15 \%$ of total features) are detected under the criterion of the corrected $p$-value (Students t-test) $\leq 0.05$, fold change $\geq$ 1.5 or $\leq 0.667$ and VIP $>=1$. The result is comparable with that of the previous study [42].

For multivariate analysis, PCA, PLS-DA and OPLSDA are performed by metaX. In PCA analysis, the normalized peak intensity matrix is glog transformed, followed by Pareto scaling and centering, and then two components are selected. The PCA score and loading plots are shown in Fig. 6a and b, respectively. The score plot indicates that there is an apparent difference between the disease and health groups. For PLS-DA and OPLS-DA, the normalized peak intensity matrix is also glog transformed, followed by Pareto scaling and centering. Two components are selected for PLS-DA and two components (one orthogonal and one predictive) for OPLS-DA. The score and loading plots for PLS-DA and OPLS-DA are shown in Fig. 7a and c, respectively. The $R^{2} Y$ and $Q^{2} Y$ values of the PLS-DA model, which are
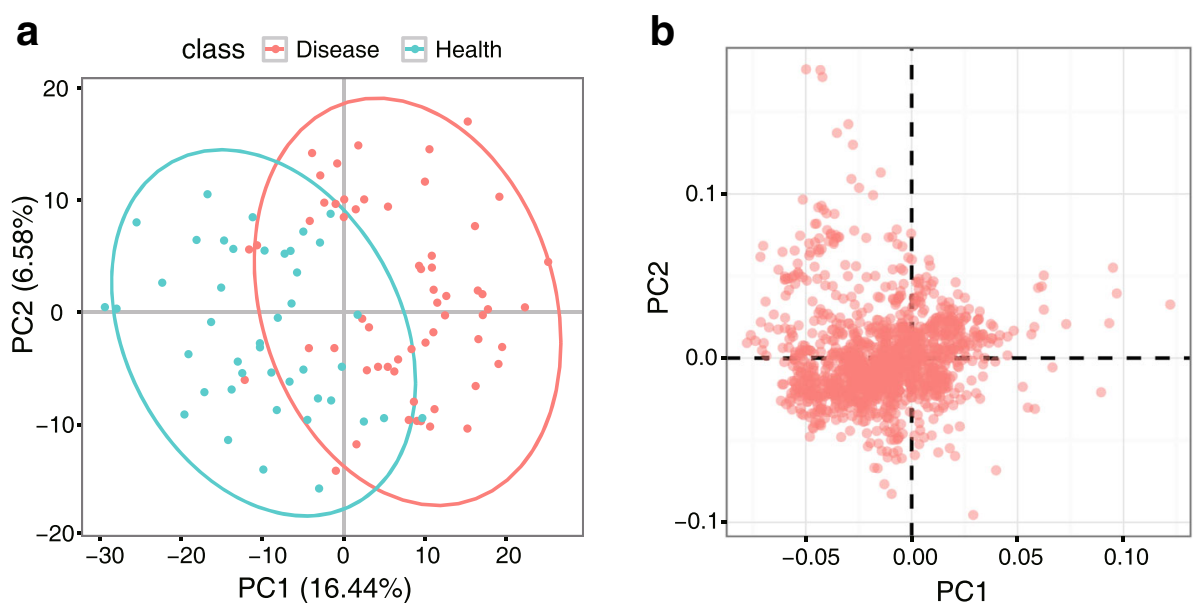

Fig. 6 The score and loading plots of PCA. a Score plot of PCA and (b) Loading plot of PCA. The different points in the figures refer to different samples, and the samples are color-coded according to their group information. The QC samples were removed before performing the PCA analysis 

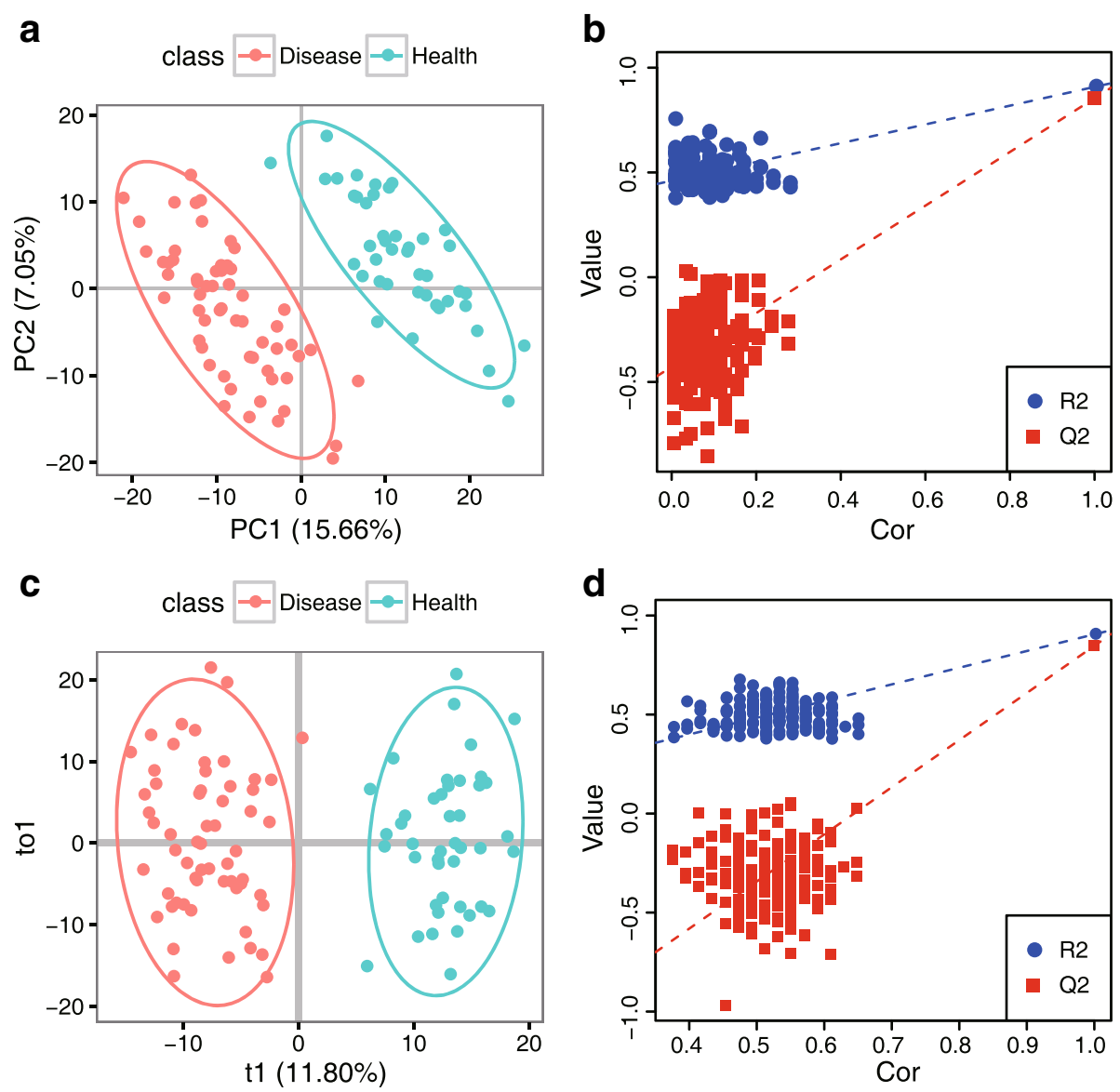

Fig. 7 The score and permutation test plots of PLS-DA and OPLS-DA. a Score plot of PLS-DA. $R^{2} Y: 0.908, Q^{2} Y: 0.854$. b Permutation test plot of PLS-DA, $p$-value $<=0.05$. c Score plot of OPLS-DA. $R^{2} Y: 0.905, Q^{2} Y: 0.847$. d Permutation test plot of OPLS-DA, $p$-value $<=0.05$. The different points in the score plots (A and C) refer to different samples, and the samples are color-coded according to their group information. The number of permutations for the permutation test is 200

0.908 and 0.854 , respectively, indicate that the model has good goodness of fit and predictive ability. The $R^{2} Y$ and $\mathrm{Q}^{2} \mathrm{Y}$ values of the OPLS-DA model, which are 0.905 and 0.847 , respectively, indicate that the model also has good goodness of fit and predictive ability. Overall, the two multivariate data analysis methods, PLS-DA and OPLS-DA, give similar results. To test the validity of the models of PLS-DA and OPLS-DA, a permutation test ( $n$ $=200$ ) is performed. As shown in Fig. $7 \mathrm{~b}$ and d, the test indicated that the two models are valid, and the good predictive ability of the model is not because of over-fitting with a $p$-value less than 0.05 . Taken together, the results of PCA and PLS-DA (or OPLS-DA) show a distinct separation between the disease and health groups.

Table 3 The biomarkers selected by metaX

\begin{tabular}{lclllll}
\hline MZ & RT $(\mathrm{min})$ & Mass & HMDB & Name & Delta (ppm) & Chemical formula \\
\hline 308.0498 & 10.46 & 285.0629 & HMDB14387 & Cladribine & -8.18 & C10H12CIN5O3 \\
424.3412 & 11.94 & 423.3349 & HMDB06469 & Linoleyl carnitine & -2.31 & C25H45NO4 \\
155.0281 & 2.81 & 116.066 & HMDB32411 & 2-Methyl-1-methylthio-2-butene & -8.77 & C6H12S \\
130.0499 & 3.43 & 129.0426 & HMDB00267 & Pyroglutamic acid & 0.15 & C5H7NO3 \\
174.9913 & 2.30 & NULL & NULL & NULL & NULL & NULL \\
309.0533 & 10.47 & 270.0892 & HMDB33940 & Vignafuran & 3.44 & C16H14O4 \\
425.3446 & 11.94 & 424.3341 & HMDB06327 & Alpha-Tocotrienol & 7.62 & C29H44O2 \\
324.0443 & 9.33 & 301.0563 & HMDB01062 & N-Acetyl-D-Glucosamine 6-Phosphate & -3.86 & C8H16NO9P \\
\hline
\end{tabular}




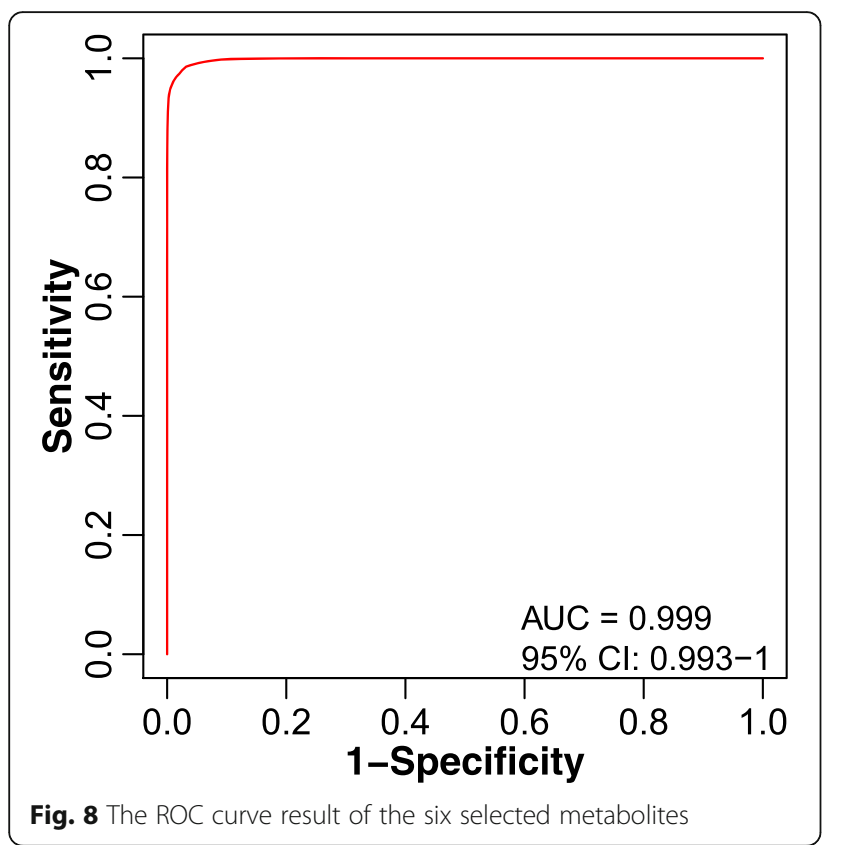

Biomarker analysis, metabolite identification and pathway analysis

To create the classification model between the disease and health groups, the functions implemented in metaX are used to conduct the biomarker selection, model creation and performance evaluation. A recursive feature elimination algorithm with the random forest model is used to select the best feature set. During the treatment, 5-fold crossvalidation is used to optimize the model and reduce overfitting. As shown in Table 3, 8 features were selected. To further evaluate the performance of the 8 selected features, the 102 samples were randomly split into two sample sets. One sample set (Disease: 29, Health 29) was for model building and the other (Disease: 14, Health 30) was for testing. Based on the two data sets, the 8 features were used to build a random forest model, and a receiver operating characteristic (ROC) curve of this model was plotted and is shown in Fig. 8. The result indicated that the model based on the 8 features had a good result with an area under the ROC (AUROC) curve of 0.999. The 8 features were then identified based on the HMDB (version 3.6) database

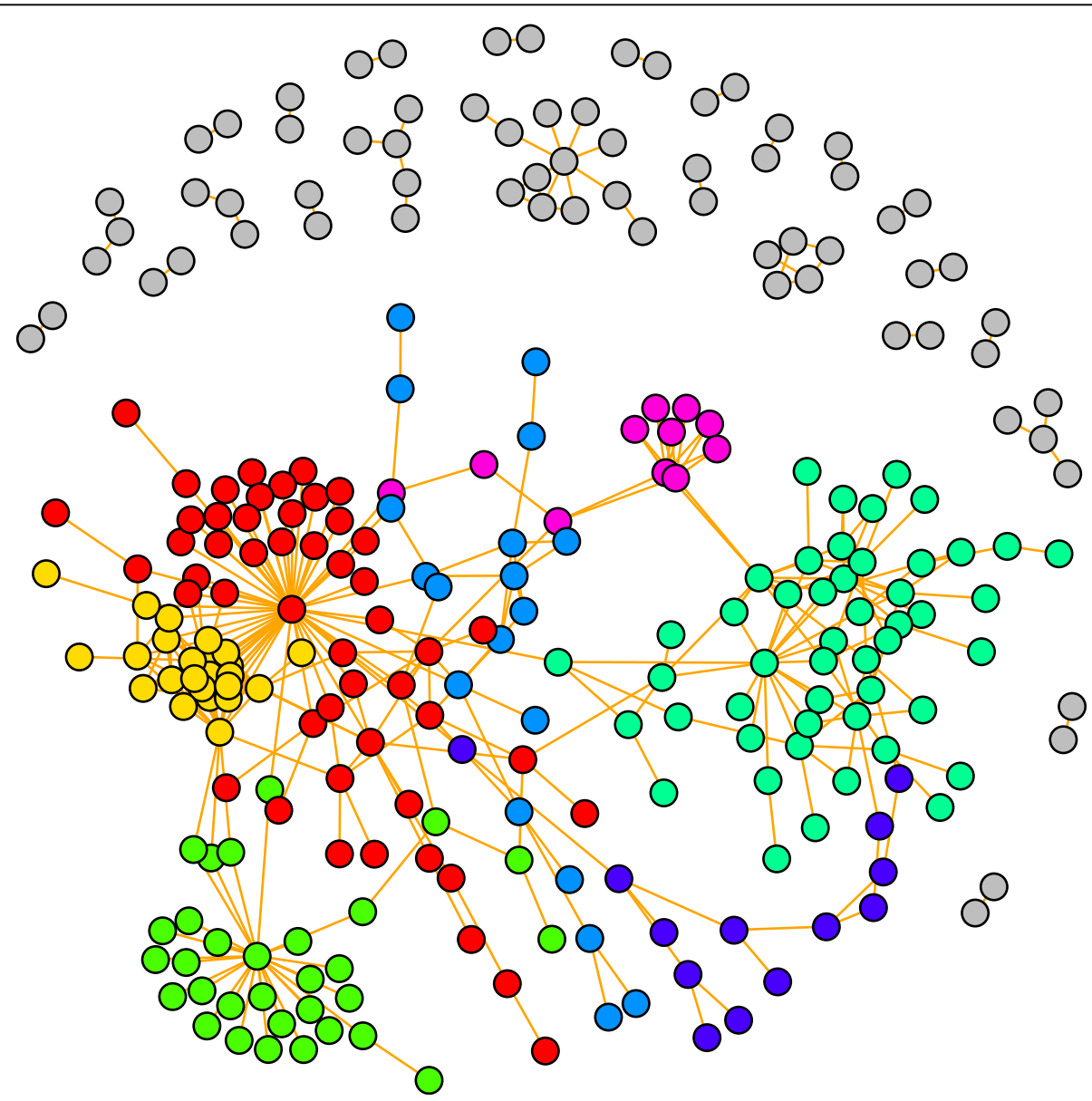

Fig. 9 The differential correction network. The top six largest numbers of nodes communities were color-coded. Detailed information about the samples and their communities are presented in Table S3 
through metaX. Seven out of the 8 features were identified with a mass accuracy of $<10 \mathrm{ppm}$ (parts per million). The putative identified metabolites were then submitted to the IMPaLA website (version 9) through metaX to perform the pathway analysis, and the results are presented in Additional file 2: Table S2.

\section{Correlation network analysis}

Network-based correlation analysis is a complementary method to the traditional univariate and multivariate statistics that is taken in metabolomics analysis to identify metabolite changes in response to variable status of physiology. All of the features with the normalized intensity described above were used to perform the differential correction network analysis. This analysis can be used to detect the interconnection of metabolite pairs whose relationships are significantly altered due to the disease process. In this study, only the metabolite pairs that had significant differential correlations ( $q$-value $<=0.01$ ) between the disease and health populations were used to build the network. As shown in Fig. 9, of the network with 266 nodes and 444 edges, a giant component $(198 / 266,74.44 \%)$ was found and the community detection analysis using the fast greedy modularity optimization algorithm against this component resulted in seven communities, in which each one has equal to or greater than 10 nodes detected. In addition, metaX can estimate three centrality metrics (degree, closeness and betweenness) for each node, and they reflect the importance of the node in the entire network (Additional file 3: Table S3). Differentially correlation network analysis is expected to provide useful insights into the underlying biological processes of the clinical development of CHD.

\section{Conclusions}

metaX presents a complete data processing software that is easy to operate and capable of dealing with large-scale metabolomics datasets. A metaX user can customize the pipeline according to the research requirements. Compared to software for metabolomics datasets that requires highmanual interaction, metaX requires much less manual interaction and can be used in a command line or webbased user-friendly interface. Based upon the fast process and the optimized workflow, therefore, metaX would greatly improve the interpretation of metabolomics data.

\section{Additional files}

Additional file 1: Table S1. The fold change and $p$-value for all of the features. (XLSX $146 \mathrm{~kb}$ )

Additional file 2: Table S2. The pathway analysis results for the 8 selected biomarkers. (XLSX 14 kb)

Additional file 3: Table S3. The centrality metrics for each node in the network. (XLSX $22 \mathrm{~kb})$

\section{Abbreviations}

FDR: False discovery rate; GUI: Graphical user interface; HMDB: Human metabolome database; MS/MS: Tandem mass spectrometry; OPLSDA: Orthogonal partial least squares discriminant analysis; PCA: Principal component analysis; PLS-DA: Partial least square discriminant analysis; QC: Quality control; ROC: Receiver operating characteristic

Acknowledgements

We thank Dr. David Broadhurst for the implementation of QC-RSC method.

\section{Funding}

This study was supported in part by the International Science \& Technology Cooperation Program of China (2014DFB30020), Chinese National Basic Research Programs (2014CBA02002-A, 2014CBA02005) and the National High-Tech Research and Development Program of China (2012AA020202). The funding body was not involved in the design of the study and collection, analysis, and interpretation of data or in writing the manuscript.

Availability of data and materials

GPL-2 licensed and available in the Bioconductor framework.

- Project name: metaX software

- Project home page: http://metax.genomics.cn/

- Operating system(s): Linux, Mac OSX, Windows

- Programming language: R, JAVA

- Other requirements: None

- License: GPL-2

- Any restrictions to use by non-academics: GPL-2

\section{Authors' contributions}

BW conceived of and designed the project. BW and CWZ wrote the R package. BW, MZL and CWZ developed the website of metaX. BW, MZL and CWZ performed the data analysis and tested the software. BW and SQL wrote the paper and all authors revised and approved.

\section{Competing interests}

The authors declare that they have no competing interests.

Consent for publication

Not applicable.

Ethics approval and consent to participate Not applicable.

\section{Publisher's Note}

Springer Nature remains neutral with regard to jurisdictional claims in published maps and institutional affiliations.

Received: 14 October 2016 Accepted: 3 March 2017

Published online: 21 March 2017

\section{References}

1. Rochfort S. Metabolomics Reviewed: A New "Omics" Platform Technology for Systems Biology and Implications for Natural Products Research. J Nat Prod. 2005;68(12):1813-20.

2. Gowda GAN, Djukovic D. Overview of Mass Spectrometry-Based Metabolomics. Oppor Challenges. 2014;1198:3-12.

3. Frédérich $M$, Pirotte $B$, Fillet $M$, de Tullio $P$. Metabolomics as a Challenging Approach for Medicinal Chemistry and Personalized Medicine. J Med Chem. 2016;59(19):8649-66.

4. Smith CA, Want EJ, O'Maille G, Abagyan R, Siuzdak G. XCMS: processing mass spectrometry data for metabolite profiling using nonlinear peak alignment, matching, and identification. Anal Chem. 2006;78(3):779-87.

5. Kuhl C, Tautenhahn R, Bottcher C, Larson TR, Neumann S. CAMERA: an integrated strategy for compound spectra extraction and annotation of liquid chromatography/mass spectrometry data sets. Anal Chem. 2012;84(1): 283-9.

6. Fernandez-Albert F, Llorach R, Andres-Lacueva C, Perera A. An R package to analyse LC/MS metabolomic data: MAIT (Metabolite Automatic Identification Toolkit). Bioinformatics. 2014;30(13):1937-9. 
7. Xia J, Psychogios N, Young N, Wishart DS. MetaboAnalyst: a web server for metabolomic data analysis and interpretation. Nucleic Acids Res. 2009; 37(Web Server issue):W652-60.

8. Giacomoni F, Le Corguille G, Monsoor M, Landi M, Pericard P, Petera M, Duperier C, Tremblay-Franco M, Martin JF, Jacob D, et al. Workflow4Metabolomics: a collaborative research infrastructure for computational metabolomics. Bioinformatics. 2015;31(9):1493-5.

9. Di Guida R, Engel J, Allwood JW, Weber RJ, Jones MR, Sommer U, Viant MR, Dunn WB. Non-targeted UHPLC-MS metabolomic data processing methods: a comparative investigation of normalisation, missing value imputation, transformation and scaling. Metabolomics. 2016;12:93.

10. Engskog MKR, Haglöf J, Arvidsson T, Pettersson C. LC-MS based global metabolite profiling: the necessity of high data quality. Metabolomics. 2016; 12(7):1-9.

11. Team RC. R: A Language and Environment for Statistical Computing. Vienna: R Foundation for Statistical Computing; 2016.

12. Ernest B, Gooding JR, Campagna SR, Saxton AM, Voy BH. MetabR: an R script for linear model analysis of quantitative metabolomic data. BMC Res Notes. 2012;5:596.

13. Huber W, Carey VJ, Gentleman R, Anders S, Carlson M, Carvalho BS, Bravo HC, Davis S, Gatto L, Girke T, et al. Orchestrating high-throughput genomic analysis with Bioconductor. Nat Methods. 2015;12(2):115-21.

14. Dunn WB, Broadhurst D, Begley P, Zelena E, Francis-Mclntyre S, Anderson N, Brown M, Knowles JD, Halsall A, Haselden JN, et al. Procedures for largescale metabolic profiling of serum and plasma using gas chromatography and liquid chromatography coupled to mass spectrometry. Nat Protoc. 2011;6(7):1060-83.

15. Gromski PS, Xu Y, Kotze HL, Correa E, Ellis DI, Armitage EG, Turner ML, Goodacre R. Influence of missing values substitutes on multivariate analysis of metabolomics data. Metabolites. 2014;4(2):433-52.

16. van den Berg RA, Hoefsloot HC, Westerhuis JA, Smilde AK, van der Werf MJ. Centering, scaling, and transformations: improving the biological information content of metabolomics data. BMC Genomics. 2006;7:142.

17. Edmands WM, Barupal DK, Scalbert A. MetMSLine: an automated and fully integrated pipeline for rapid processing of high-resolution LC-MS metabolomic datasets. Bioinformatics. 2015;31(5):788-90.

18. Dunn WB, Wilson ID, Nicholls AW, Broadhurst D. The importance of experimental design and QC samples in large-scale and MS-driven untargeted metabolomic studies of humans. Bioanalysis. 2012;4(18):2249-64.

19. Kirwan JA, Broadhurst DI, Davidson RL, Viant MR. Characterising and correcting batch variation in an automated direct infusion mass spectrometry (DIMS) metabolomics workflow. Anal Bioanal Chem. 2013; 405(15):5147-57

20. Shen X, Gong X, Cai Y, Guo Y, Tu J, Li H, Zhang T, Wang J, Xue F, Zhu Z-J. Normalization and integration of large-scale metabolomics data using support vector regression. Metabolomics. 2016;12(5):1-12.

21. Johnson WE, Li C, Rabinovic A. Adjusting batch effects in microarray expression data using empirical Bayes methods. Biostatistics. 2006;8(1):118-27.

22. Szymanska E, Saccenti E, Smilde AK, Westerhuis JA. Double-check: validation of diagnostic statistics for PLS-DA models in metabolomics studies. Metabolomics. 2012;8 Suppl 1:3-16.

23. Benjamini $Y$, Hochberg $Y$. Controlling the false discovery rate: a practical and powerful approach to multiple testing. J R Stat Soc Ser B Methodol. 1995;1:289-300.

24. Mevik B-H, Wehrens $\mathrm{R}$, Liland $\mathrm{KH}$ : pls: Partial least squares and principal component regression. R package version 2015;2(5). https://cran.r-project. org/web/packages/pls/index.html

25. Thévenot EA, Roux A, Xu Y, Ezan E, Junot C. Analysis of the Human Adult Urinary Metabolome Variations with Age, Body Mass Index, and Gender by Implementing a Comprehensive Workflow for Univariate and OPLS Statistical Analyses. J Proteome Res. 2015;14(8):3322-35.

26. van Iterson M, t Hoen PA, Pedotti P, Hooiveld GJ, den Dunnen JT, van Ommen GJ, Boer JM, Menezes RX. Relative power and sample size analysis on gene expression profiling data. BMC Genomics. 2009;10:439.

27. Fukushima A. DiffCorr: An R package to analyze and visualize differential correlations in biological networks. Gene. 2013;518(1):209-14.

28. Csardi G, Nepusz T. The igraph software package for complex network research. Inter J Complex Syst. 2006;1695(5):1-9.
29. Shannon P, Markiel A, Ozier O, Baliga NS, Wang JT, Ramage D, Amin N, Schwikowski B, Ideker T. Cytoscape: a software environment for integrated models of biomolecular interaction networks. Genome Res. 2003;13(11): 2498-504.

30. Bastian M, Heymann S, Jacomy M. Gephi: an open source software for exploring and manipulating networks. ICWSM. 2009;8:361-2.

31. Wishart DS, Jewison T, Guo AC, Wilson M, Knox C, Liu Y, Djoumbou Y Mandal R, Aziat F, Dong E, et al. HMDB 3.0-The Human Metabolome Database in 2013. Nucleic Acids Res. 2013;41(Database issue):D801-7.

32. Kanehisa M, Goto S. KEGG: kyoto encyclopedia of genes and genomes. Nucleic Acids Res. 2000;28(1):27-30

33. Kanehisa M, Goto S, Sato Y, Furumichi M, Tanabe M. KEGG for integration and interpretation of large-scale molecular data sets. Nucleic Acids Res. 2011;40(D1):D109-14.

34. Horai H, Arita M, Kanaya S, Nihei Y, Ikeda T, Suwa K, Ojima Y, Tanaka K, Tanaka S, Aoshima K, et al. MassBank: a public repository for sharing mass spectral data for life sciences. J Mass Spectrom. 2010;45(7):703-14.

35. Wang Y, Xiao J, Suzek TO, Zhang J, Wang J, Bryant SH, Web S. PubChem: a public information system for analyzing bioactivities of small molecules. Nucleic Acids Res. 2009;37(Web Server):W623-33.

36. Sud M, Fahy E, Cotter D, Brown A, Dennis EA, Glass CK, Merrill Jr AH, Murphy RC, Raetz CR, Russell DW, et al. LMSD: LIPID MAPS structure database. Nucleic Acids Res. 2007;35(Database issue):D527-32.

37. Caspi $R$, Altman T, Billington $R$, Dreher $K$, Foerster $H$, Fulcher CA, Holland TA, Keseler IM, Kothari A, Kubo A, et al. The MetaCyc database of metabolic pathways and enzymes and the BioCyc collection of Pathway/Genome Databases. Nucleic Acids Res. 2014;42(D1):D459-71.

38. Kamburov A, Cavill R, Ebbels TM, Herwig R, Keun HC. Integrated pathwaylevel analysis of transcriptomics and metabolomics data with IMPaLA. Bioinformatics. 2011;27(20):2917-8.

39. Kuhn M. Building predictive models in R using the caret package. J Stat Softw. 2008;28(5):1-26.

40. Breiman L. Random forests. Mach Learn. 2001:45(1):5-32.

41. Gehlenborg N, Noble MS, Getz G, Chin L, Park PJ. Nozzle: a report generation toolkit for data analysis pipelines. Bioinformatics. 2013;29(8): 1089-91.

42. Feng Q, Liu Z, Zhong S, Li R, Xia H, Jie Z, Wen B, Chen X, Yan W, Fan Y, et al. Integrated metabolomics and metagenomics analysis of plasma and urine identified microbial metabolites associated with coronary heart disease. Sci Rep. 2016;6:22525.

43. Feng Q, Liu Z, Zhong S, Li R, Xia H, Jie Z, Wen B, Chen X, Yan W, Fan Y, Dryad Data $R$, et al. Data from: Integrated metabolomics and metagenomics analysis of plasma and urine identified microbial metabolites associated with coronary heart disease, Dryad Data Repository. 2016.

44. Kessner D, Chambers M, Burke R, Agus D, Mallick P. ProteoWizard: open source software for rapid proteomics tools development. Bioinformatics. 2008;24(21):2534-6.

45. Chawade A, Alexandersson E, Levander F. Normalyzer: a tool for rapid evaluation of normalization methods for omics data sets. J Proteome Res. 2014:13(6):3114-20.

\section{Submit your next manuscript to BioMed Central and we will help you at every step:}

- We accept pre-submission inquiries

- Our selector tool helps you to find the most relevant journal

- We provide round the clock customer support

- Convenient online submission

- Thorough peer review

- Inclusion in PubMed and all major indexing services

- Maximum visibility for your research

Submit your manuscript at www.biomedcentral.com/submit 